\title{
Influencia de la concentración de sustrato sobre la cinética del proceso de biometanización del alpechín, en biorreactores de lecho fluidizado con microorganismos inmovilizados.
}

\author{
Por R. Borja Padilla (1), A. Martín Martín (2) y J. A. Fiestas Ros de Ursinos (1).
}

(1) Instituto de la Grasa y sus Derivados (C.S.I.C.) Avda. Padre García Tejero n. 4. 41012 - SEVILLA

(2) Dpto. de Ingeniería Química. Facultad de Ciencias. Avda. S. Alberto Magno s/n. 14004 - CORDOBA

\section{RESUMEN}

Influencia de la concentración de sustrato sobre la cinética del proceso de biometanización del alpechín, en biorreactores de lecho fluidizado con microorganismos inmovilizados.

Se ha efectuado un estudio cinético del proceso de fermentación anaerobia del alpechín en biorreactores de lecho fluidizado que contienen en suspensión soportes de tipo arcilloso sobre los que se han inmovilizado los microorganismos responsables del proceso.

Se utilizan cuatro concentraciones de alpechin (10\%, 20\%, $40 \%$ y $80 \%$ ) para estudiar la influencia de la concentración de sustrato sobre las constantes biocinéticas del proceso en régimen continuo, estudiándose al mismo tiempo la influencia del soporte de inmovilización sobre las mismas.

Para ello, se aplica el modelo de Chen-Hashimoto de producción de metano, obteniéndose los valores de los parámetros cinéticos $\mu_{\max }$ y $\mathrm{K}$, para cada uno de los casos estudiados.

Se obtiene una disminución considerable de los valores de estos parámetros al aumentar la concentración de alpechín utilizada como influente, si bien este descenso de las constantes cinéticas varia según el soporte considerado

PALABRAS-CLAVE: Alpechín - Biometanización - Cinética - Inmovilización - Soporte.

\section{SUMMARY}

Influence of substrate concentration on the kimetics of olive mill wastewater biomethanation in fluid bed bioreactors using inmobilized microorganisms.

A kinetic study has been made of the anaerobic olive mill wastewater fermentation process in fluid bed bioreactors containing clay supports in suspension, on which the microorganisms responsible for the process have been inmobilized.

Four concentrations of wastewater have been used $(10 \%$, $20 \%, 40 \%$ and $80 \%$ ) to study the influence of the substrate concentration, and at the same time, of the immobilization support, on the biokinetic constants of the continuous process.

The Chen-Hashimoto model of methane production has been applied, obtaining the values of the kinetic parameters $\mu_{\max }$ and $K$ for each case studied.

The values of these parameters decreased considerably with increasing concentration of influent wastewater, though this decrease varied depending on the support used.

KEY-WORS: Biomethanation - Inmobilization - Kinetics Olive mill wastewater - Support

\section{INTRODUCCION}

El alpechín, nombre que generalmente se asigna al agua residual de la industria de obtención del aceite de oliva, está constituido principalmente por el agua de vegetación de la aceituna, así como por pequeñas porciones de tejidos blandos de la pulpa de aceituna y aceite, juntamente con el agua utilizada en el proceso, constituyendo todo ello una emulsión relativamente estable.

Según el sistema empleado: almazara clásica - continua, difiere la composición del alpechín, ya que mientras en los sistemas clásicos de capachos la adición de agua es muy pequeña o nula, en los sistemas continuos se utiliza del orden de 1 litro de agua por kilogramo de aceitunas a molturar, por lo que su poder contaminante es muy diferente (1).

Este gran poder contaminante, en base a su elevada demanda química y biológica de oxígeno, junto con sus elevados volúmenes de producción en la Comunidad Andaluza y en la Cuenca del Mediterráneo en general, ocasionan un problema medioambiental de gran magnitud (2).

De entre las posibles soluciones y tecnologías disponibles de cara a la depuración y aprovechamiento del alpechín, destaca la fermentación anaerobia o biometanización cuyas ventajas quedaron resaltadas en un trabajo precedente (1).

Dada la pequeña velocidad de reproducción de los microorganismos anaerobios se han desarrollado diversas tecnologías para su inmovilización en el seno de los biorreactores, con el fin de evitar su pérdida con los efluentes y con ello una posible disminución de la velocidad del proceso.

Entre los biorreactores más utilizados para llevar a cabo este proceso destacan los de lecho fluidizado, en los que las bacterias colonizan particulas de determinados materiales, aumentando la 
superficie utilizable para el crecimiento bacteriano. Por otra parte, estos reactores son los que permiten conseguir cargas volumétricas más altas, superiores a los $50 \mathrm{~kg} \mathrm{DQO} / \mathrm{m}^{3} \mathrm{~d}$, lo que los hace muy adecuados para el tratamiento de aguas residuales de alta carga orgánica, como la que nos ocupa (3).

Los resultados obtenidos en investigaciones anteriores sobre microbiología y bioquímica de los procesos anaerobios pusieron de manifiesto la influencia que sobre este proceso ejercen diferentes soportes utilizados para la inmovilización de los microorganismos responsables del mismo (4) (5) (6) (7)

El objetivo de este trabajo es el de obtener las constantes cinéticas que rigen el proceso continuo de biometanización del alpechín en biorrectores de lecho fluidizado, con microorganismos inmovilizados en soportes de tipo arcilloso, estudiando por un lado la influencia de la concentración de sustrato y por otro, el efecto del soporte utilizado para la inmovilización bacteriana.

A partir de resultados obtenidos mediante experimentos de fermentación anaerobia del alpechín en régimen discontinuo, se seleccionan los soportes Montmorillonita y Sepiolita (Pansil), por ser los más prometedores de todos los ensayados previamente (2).

\section{PARTE EXPERIMENTAL.}

\subsection{Equipo experimental.}

Los reactores están constituidos por unidades de digestión anaerobia (U. D. A.) (1) a los que se acopla una pieza troncocónica de 1,5 litros de capacidad para facilitar por una parte la expansión y fluidización de la biomasa y por otra su decantación, con el fin de evitar su pérdida con el efluente. La pieza lleva acoplada una boca central superior para la salida del biogás y una lateral para la salida del efluente.

La expansión y fluidización de la biomasa se realiza con un sistema de agitación magnética. Todo este equipo se introduce en una cámara termostatizada a la temperatura de $35^{\circ} \mathrm{C}$.

La alimentación se realiza por la parte inferior del reactor mediante una bomba peristáltica.

El biogás producido se pasa a través de una disolución de $\mathrm{NaOH}$ para eliminar el $\mathrm{CO}_{2}$ y se determina el volumen de metano producido en función del tiempo (1).
La salida de efluente se realiza a través de un cierre hidráulico con una columna de líquido de 25 $\mathrm{cm}$ de altura y $50 \mathrm{ml}$ de volumen para evitar la entrada de aire al reactor y la salida del biogás.

\subsection{Soportes utilizados.}

Los materiales utilizados como soporte para la inmovilización de las bacterias responsables del proceso son: Montmorillonita y Sepiolita (Pansil) (1).

\subsection{Inóculo.}

Como inóculo para el arranque de los digestores se utilizan lodos procedentes de una balsa de vertido de alpechín debidamente diluidos y neutralizados (1).

\subsection{Características del alpechín utilizado.}

Las características del alpechín utilizado se resumen en la tabla I.

Tabla 1

Características del alpechín utilizado.

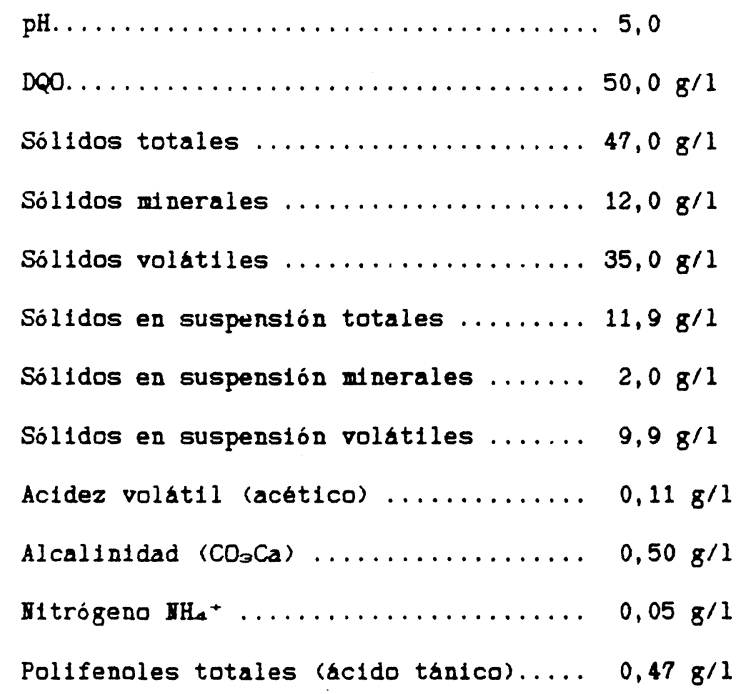

\subsection{Procedimiento experimental.}

\section{Etapa preliminar.}

Se utilizan dos unidades de digestión anaerobia, una de las cuales contine como soporte Montmorillonita y otra Sepiolita (Pansil). A cada una de ellas, se adiciona inicialmente $750 \mathrm{ml}$ de agua 
destilada, $250 \mathrm{ml}$ del inóculo ya mencionado y 10 gramos del soporte correspondiente.

Previa a la realización de los experimentos en régimen continuo, los digestores se someten a un periodo de aclimatación, añadiéndose cargas volumétricas sucesivas de alpechín, realizándose seguidamente una serie de experimentos en discontinuo, con alimentaciones progresivas de este agua residual.

Tras esta etapa inicial, se acopla a los reactores una pieza troncocónica, para facilitar la expansión, fluidización y decantación de la biomasa bacteriana y se realiza el montaje experimental anteriormente descrito.

\section{Ejecución de los experimentos.}

Se realizan cuatro series de experimentos, en régimen continuo en los que se utilizan concentraciones del $10 \%, 20 \%, 40 \%$ y $80 \%$ de alpechín como influentes, trabajándose en cada una de ellas, con caudales de alimentación de $25,35,40,50,60$ y $100 \mathrm{ml}$ influente / día, que corresponden a 44,8 , $32,0,28,0,22,4,18,6$ y 11,2 días de tiempo de retención hidráulica respectivamente, teniendo en cuenta que el reactor, tiene un volumen de 1,12 litros.

Para cada caudal de alimentación o tiempo de retención hidráulica correspondiente, se determina tras alcanzarse condiciones de estado estacionario el volumen de metano producido.

\subsection{Técnicas analíticas.}

Los análisis se realizan de acuerdo con el "Standard Methods for the Examination of Water and Wastewater" (8).

\section{RESULTADOS EXPERIMENTALES.}

En las tablas II y III se expresan los valores del volumen de metano (ml) obtenidos en función del caudal utilizado (ml/día) para los influentes con concentraciones de alpechín de 10\%, 20\%, 40\% y $80 \%$ en los reactores con soporte Montmorillonita y Pansil, respectivamente.

\section{DISCUSION DE LOS RESULTADOS.}

Para estudiar la influencia de la concentración de sustrato y del soporte utilizado, sobre las cons-
Tabla \|

Volumen de metano (mililitros) en función del caudal (mililitros/día) para las distintas concentraciones de alpechín utilizadas como influente. Reactor con soporte Montmorillonita.

\begin{tabular}{|c|c|c|c|c|c|c|}
\hline Concentración & & & & audal & & \\
\hline & 25 & 35 & 40 & 50 & 60 & 100 \\
\hline $10 \%$ & 27 & 37 & 41 & 49 & 57 & 83 \\
\hline $20 \%$ & 52 & 70 & 79 & 93 & 110 & 168 \\
\hline $40 \%$ & 110 & 150 & 168 & 204 & 240 & 380 \\
\hline $80 \%$ & 285 & 378 & 424 & 524 & 616 & 976 \\
\hline
\end{tabular}

Tabla III

Volumen de metano (mililitros) en función del caudal (mililitros/dia) para las distintas concentraciones de alpechin utilizadas como influente. Reactor con soporte Pansil.

Concentración

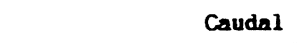

\begin{tabular}{|c|c|c|c|c|c|}
\hline & 25 & 35 & 40 & 50 & 60 \\
\hline $10 \%$ & 27 & 36 & 40 & 49 & 56 \\
\hline $20 \%$ & 61 & 81 & 90 & 105 & 125 \\
\hline $0 \%$ & 119 & 160 & 178 & 215 & 252 \\
\hline & 290 & 392 & 440 & 536 & 629 \\
\hline
\end{tabular}

tantes biocinéticas del proceso continuo de biometanización del alpechín se realizan una serie de experimentos en los que se utilizan distintas concentraciones de este agua residual como influente en dos reactores anaerobios de lecho fluidizado, con los que se trabaja a distintos caudales de alimentación y por tanto a distintos tiempos de retención hidráulica.

Entre los factores que más influyen sobre el proceso de digestión anaerobia destaca el pH del medio, por lo que resulta de gran interés su seguimiento a lo largo del proceso. Este parámetro es función de la alcalinidad del sistema, de la presión parcial del $\mathrm{CO}_{2}$ y de la concentración de ácidos volátiles que existan en el medio. Para obtener buenos rendimientos de degradación de materia orgánica y de producción de metano, el $\mathrm{pH}$ debe encontrarse entre 6,8 y 8,0 . Para estos experimentos, este parámetro se mantiene dentro de estos límites óptimos oscilando entre los valores 7,2 y 7,6, sin acusar efectos significativos por variaciones del caudal y de la concentración. 


\subsection{Modelo de Chen-Hashimoto para proce- sos de digestión anaerobia.}

Chen y Hashimoto (9) proponen un modelo cinético, en el que se parte de la ecuación cinética de Contois, para definir la velocidad específica de crecimiento de microorganismos.

$$
\mu=\frac{\mu_{\max } \cdot S}{B \cdot X+S}
$$

donde:

$\mu$, es la velocidad específica de crecimiento de microorganismos.

$\mu_{\max }$, la velocidad máxima específica de crecimiento microbiano.

$S$, la concentración de sustrato.

$X$, la concentración de masa celular.

$B$, es un parámetro cinético adimensional, cuyo valor es igual al cociente entre la concentración de sustrato y la de masa celular, que da lugar a una velocidad específica de crecimiento, igual a la mitad de su velocidad máxima.

En digestores de mezcla completa y flujo continuo, las velocidades de cambio de masa celular y de concentración de sustrato, vienen expresadas por las siguientes ecuaciones:

$$
\frac{d X}{d t}=\mu \cdot X-\frac{X}{\theta}
$$

donde: $\frac{d S}{d t}=-r+\frac{\text { So-S }}{\theta}$

$r=$ velocidad de utilización de sustrato.

$\theta=$ tiempo de retención hidráulico.

So $=$ concentración de sustrato en el influente.

$S=$ concentración de sustrato en el efluente.

La relación entre $r$ y $\mu$ viene dada por la siguiente ecuación:

$$
\mu=\left(Y_{x / s}\right) \cdot r
$$

siendo $Y_{x / s}$ el coeficiente de rendimiento (masa celular/ masa sustrato).

En este modelo, el coeficiente de rendimien- to se considera constante y no se tiene en cuenta el fenómeno de muerte celular.

En estado estacionario, $\mathrm{dX} / \mathrm{dt}=\mathrm{O}$ y $\mathrm{dS} / \mathrm{dt}=\mathrm{O}$, con lo cual

$$
\begin{aligned}
& \mu=1 / \theta \\
& r=(\text { So }-S) / \theta \\
& X=Y_{x / s}(\text { So }-S)
\end{aligned}
$$

Al ser la concentración másica celular difícil de medir se elimina en las ecuaciones en que aparece utilizando la relación (7); sustituyendo en la ecuación de Contois (ecuación 1), se obtiene:

$$
\frac{\mu}{\mu_{\max }}=\frac{\mathrm{S} / \mathrm{So}}{\mathrm{K}+(1-\mathrm{K}) \cdot(\mathrm{S} / \mathrm{So})}
$$

donde:

$\mathrm{K}$ = parámetro cinético adimensional igual a $\mathrm{Y}_{\mathrm{x} / \mathrm{s}}$ multiplicado por $B$.

El tiempo mínimo de retención que indica donde se produce el efecto de lavado de microorganismos, es numéricamente igual al recíproco de la velocidad máxima de crecimiento.

$$
\theta_{\min }=1 / \mu_{\max }
$$

sustituyendo la ecuación (5) en la (8), queda:

$$
\mathrm{S} / \mathrm{S}_{\mathrm{o}}=\mathrm{K} /\left(\mu_{\max } \cdot \theta-1+\mathrm{K}\right)
$$

Por otra parte, la producción de metano que se genera en un proceso anaerobio, es directamente proporcional a la eliminación de sustrato, ya que otras formas de eliminación del mismo hacia sulfhídrico e hidrógeno son poco significativas (9).

Por esta razón, en un sistema de este tipo, la concentración de sustrato que se introduce en el digestor, es directamente proporcional a $\mathrm{B}$; y la concentración de sustrato que queda en el digestor en cada momento a $\left(B_{0}-B\right)$, donde:

$\mathrm{B}=$ litros de metano producido en condiciones normales de presión y temperatura por gramo de sustrato añadido.

$\mathrm{B}_{\mathrm{o}}=$ litros de metano producido en condiciones normales de presión y temperatura por gramo de sustrato añadido a tiempo de retención infinito. 

DQO.

La concentración de sustrato se evalúa como

Por tanto, a partir de la ecuación (10) se puede escribir:

$$
\frac{B_{0}-B}{B_{0}}=\frac{K}{\left(\theta / \theta_{\min }\right)-1+K}
$$

de donde:

$$
B=B_{0}\left(1-\frac{K}{(\theta / \theta \min )-1+K}\right)
$$

La ecuación (12) indica que, cuando $\theta / \theta_{\min } \gg$ 11 - KI la representación de $B$ frente a $1 / \theta$ es una línea resta, en la que $B$ coincide con el valor de $B$ cuando $\theta$ tiende a infinito.

Este método se utiliza para determinar el valor de $B_{0}$.

Reordenando la ecuación (11), se obtiene:

$$
\theta=\theta_{\min }+\theta_{\min } \cdot K \cdot \frac{B}{\left(B_{0}-B\right)}
$$

La representación de $\theta$ frente a B/ (Bo - B) da lugar a una línea recta cuya ordenada en el origen es igual a $\theta_{\min }$ y su pendiente a $\mathrm{K} \cdot \theta_{\text {min }}$.

De esta forma, pueden obtenerse los parámetros cinéticos $\mathrm{K}$ y $\mu_{\max }\left(\mu_{\max }=1 / \theta_{\min }\right)$ a partir de los datos experimentales de producción de metano.

\subsection{Aplicación del modelo de Chen-Hashi- moto a los resultados experimentales.}

La velocidad específica máxima de crecimiento $\mu_{\max }$ y la constante cinética del proceso $\mathrm{K}$, se determinan a partir de la ecuación:

$$
\theta=\frac{1}{\mu_{\max }}+\frac{\mathrm{K}}{\mu_{\max }} \cdot \frac{\mathrm{B}}{\left(\mathrm{B}_{\mathrm{o}}-\mathrm{B}\right)}
$$

ecuación análoga a la (13) expuesta con anterioridad.

Sin embargo previamente han de determinarse los valores de $\mathrm{B}_{\mathrm{o}}$, haciendo uso de la ecuación (12); en este caso, se observa que cuando $\theta / \theta_{\min }$
>> $\mid 1-\mathrm{KI}$ las representaciones gráficas de $\mathrm{B}$ frente a $1 / \theta$ son líneas rectas, en las que $B$ coincide con $\mathrm{B}_{\mathrm{o}}$, cuando el tiempo de retención tiende a infinito, es decir, la ordenada en el origen de las representaciones lineales coincide con $\mathrm{B}_{0}$.

En las Tablas IV y $\mathrm{V}$ se recogen los valores de $B$ en función del tiempo de retención hidráulica para cada una de las concentraciones de alpechín utilizadas como influente en los reactores con soporte Montmorillonita y Pansil respectivamente. Estos valores se calculan teniendo en cuenta que el alpechín utilizado tiene un DQO de $50 \mathrm{~g} / \mathrm{l}$, y en consecuencia las diluciones del 10\%, 20\%, 40\% y $80 \%$ del mismo, una DQO de $5,10,20$ y $40 \mathrm{~g} /$ I respectivamente.

Tabla IV

\begin{tabular}{|c|c|c|c|c|}
\hline \multirow[t]{2}{*}{$\begin{array}{l}\text { Tlempo } \\
\text { Retenctón }\end{array}$} & \multirow[b]{2}{*}{$10 \%$} & \multicolumn{2}{|c|}{ Concentración } & \multirow[b]{2}{*}{$80 \%$} \\
\hline & & $20 \%$ & $40 \%$ & \\
\hline 44,8 & 191,3 & 184,2 & 194,8 & 252,4 \\
\hline 32,0 & 187,2 & 177,1 & 189,8 & 239,1 \\
\hline 28,0 & 181,5 & 174,9 & 186,0 & 234,7 \\
\hline 22,4 & 173,6 & 164,7 & 180,7 & 232,0 \\
\hline 18,6 & 168,3 & 162,3 & 177,1 & 227,3 \\
\hline 11,2 & 147,0 & 148,8 & 168,3 & 216,1 \\
\hline
\end{tabular}

Valores experimentales de $\mathrm{B}(\mathrm{ml} \mathrm{CH} 4 \mathrm{STP} / \mathrm{g}$ DQO) en función del tiempo de retención (días) para las distintas concentraciones de alpechín. Reactor con soporte Montmorillonita.

Tabla V

\begin{tabular}{|c|c|c|c|c|}
\hline \multirow[t]{2}{*}{$\begin{array}{c}\text { T1erpo } \\
\text { Retenc16n } \\
\end{array}$} & \multirow[b]{2}{*}{$10 \%$} & \multicolumn{2}{|c|}{ Concentración } & \multirow[b]{2}{*}{$80 \%$} \\
\hline & & $20 \%$ & $40 \%$ & \\
\hline 44,8 & 191,3 & 216,1 & 210,8 & 256,8 \\
\hline 32,0 & 182,2 & 205,0 & 202,4 & 248,0 \\
\hline 28,0 & 177,1 & 199,3 & 197,0 & 243,5 \\
\hline 22,4 & 173,6 & 186,0 & 190,4 & 237,3 \\
\hline 18,6 & 167,1 & 184,5 & 186,0 & 232,1 \\
\hline 11,2 & 141,7 & 155,0 & 169,1 & 221,4 \\
\hline
\end{tabular}

Valores experimentales de $\mathrm{B}$ ( $\mathrm{ml} \mathrm{CH} 4 \mathrm{STP} / \mathrm{g} \mathrm{DQO})$ en función del tiempo de retención (días) para las distintas concentraciones de alpechín. Reactor con soporte Pansil. 
En la Figura 1 aparecen a título de ejemplo las representaciones de $\mathrm{B}$ (litros de $\mathrm{CH}_{4}$ en condiciones normales de presión y temperatura/gramo DQO) frente a $1 / \theta\left(\right.$ días $\left.^{-1}\right)$ para el reactor con soporte
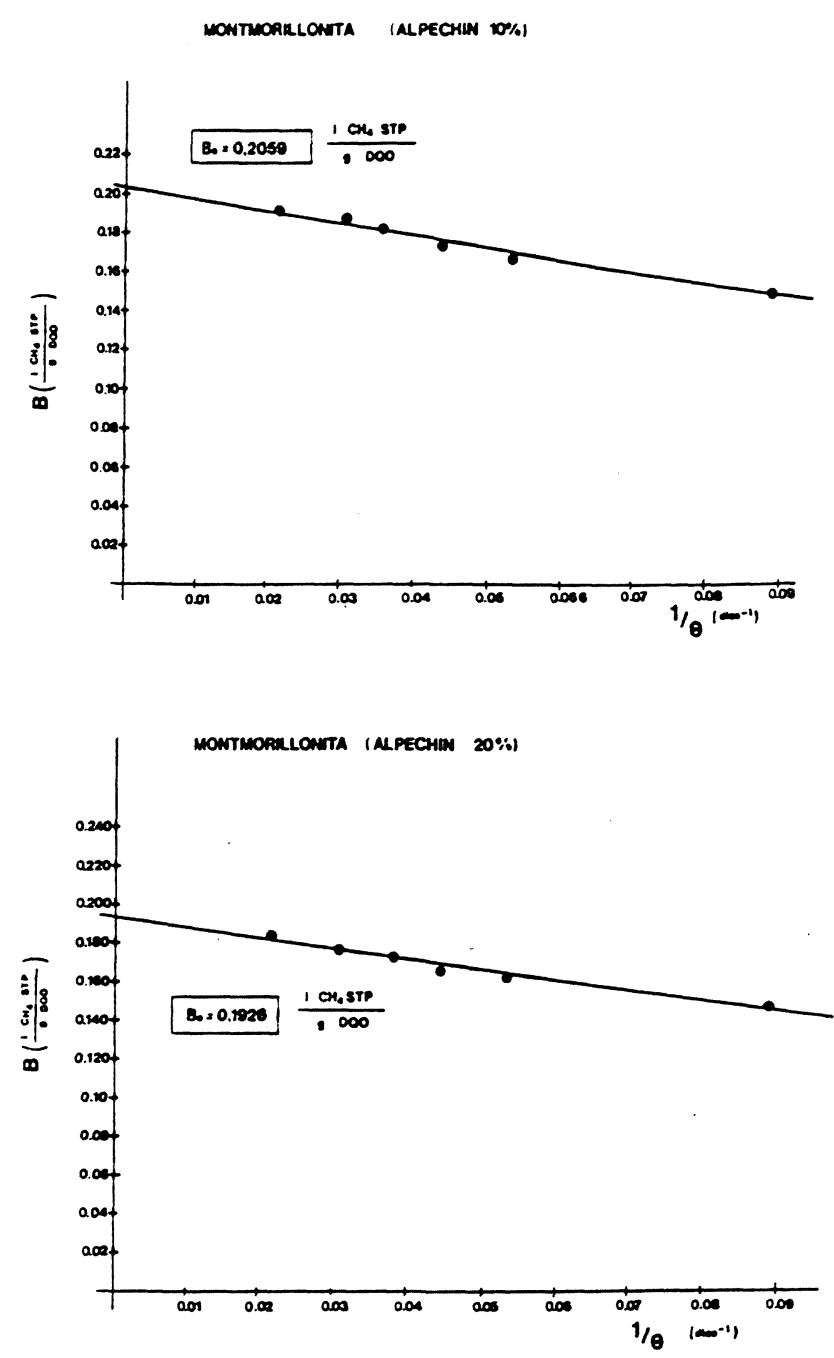

Figura 1

Efecto del tiempo de retención sobre la producción de metano, para el reactor con soporte Montmorillonita y las concentraciones del $10 \%$ y $20 \%$ de alpechín.

\section{Tabla VI}

Valores de $\mathrm{B}_{0}\left(\mathrm{ml} \mathrm{CH} \mathrm{CH}_{4}\right.$ STP/g DQO) para cada concentración de alpechín utilizada en los dos reactores considerados.

Concentractón

$\begin{array}{lllll} & 10 \% & 20 \% & 40 \% & 80 \% \\ \text { Montmorillonita } & 205,9 & 192,6 & 200,7 & 255,6 \\ \text { Pansil } & 205,1 & 231,7 & 220,2 & 263,1\end{array}$
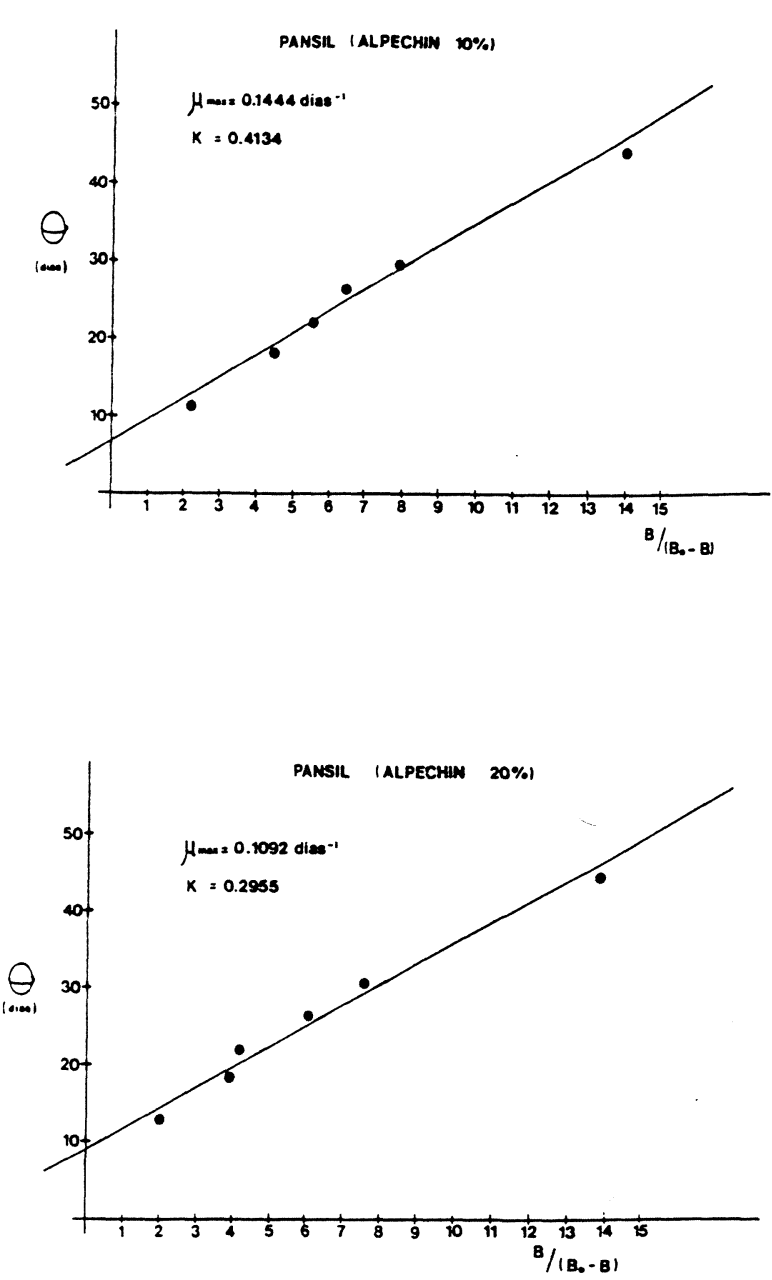

Figura 2

Determinación de los parámetros cinéticos $\mu_{\max }$ y $\mathrm{K}$ para el reactor con soporte Sepiolita (Pansil) y las concentraciones del $10 \%$ y $20 \%$ de alpechín.

Montmorillonita y las concentraciones del $10 \%$ y $20 \%$ de alpechín utilizadas cono influente.

Las parejas de valores $B, 1 / \theta$ se ajustan a una' línea recta, utilizando el método de mínimos cuadrados, de manera que la ordenada en el origen de la misma coincide con el valor de $B_{0}$. Los coeficientes de correlación obtenidos son bastante elevados en todos los casos, presentando un valor medio de 0,991 .

Los valores de $B_{0}$ obtenidos para cada una de las concentraciones de alpechín utilizadas, en cada reactor aparecen en la Tabla VI.

Una vez obtenidos los parámetros $\mathrm{B}_{0}$ (tabla $\mathrm{VI}$ ) y los valores de $B$ (tablas IV y V) a partir de los datos experimentales para cada tiempo de retención, concentración de alpechín y reactor considerado, la representación de $\theta$ frente a $B /\left(B_{0}-B\right)$ 
permite la determinación de los parámetros cinéticos $\mu_{\max }$ y $\mathrm{K}$.

En la Figura 2 se representan a modo de ejemplo los valores del tiempo de retención $\theta$ (días) frente al cociente de volúmenes de metano $B /\left(B_{0}-B\right)$ para el reactor con soporte Pansil y las concentraciones del $10 \%$ y $20 \%$ de alpechín.

Estos valores se ajustan a una línea recta, empleando el método de mínimos cuadrados, de manera que podemos obtener los valores de los parámetros cinéticos $\mu_{\max }$ y $\mathrm{K}$, a través de la ordenada en el origen y la pendiente de la recta ajustada. Así pues, teniendo en cuenta la ecuación (14):

$\mu_{\max }=1 /$ (Ordenada en el origen) .

$\mathrm{K}=$ pendiente $/$ (Ordenada en el origen) .

siendo el tiempo de retención mínimo,

$$
\theta_{\min }=1 / \mu_{\max }
$$

En las tablas VII y VIII se resumen los valores de los parámetros cinéticos $\mu_{\max }$ y $\mathrm{K}$ obtenidos en los reactores con soporte Montmorillonita y Pansil respectivamente, para las cuatro concentraciones de alpechín consideradas.

Tabla VII

Valores de los parámetros cinéticos $\mu_{\max }$ y $\mathrm{K}$ para cada concentración de alpechín. Reactor con soporte Montmorillonita.

\begin{tabular}{|c|c|c|c|c|}
\hline \multirow[t]{2}{*}{ Paránetro } & & \multicolumn{2}{|c|}{ Concentración } & \\
\hline & $10 \%$ & $20 \%$ & $40 \%$ & $80 \%$ \\
\hline$\mu_{\max }\left(\operatorname{dias}^{-1}\right)$ & 0,20 & 0,10 & 0,09 & 0,05 \\
\hline $\mathrm{K}$ & 0,59 & 0,16 & 0,10 & 0,01 \\
\hline
\end{tabular}

Tabla VIII

Valores de los parámetros cinéticos $\mu_{\max }$ y $\mathrm{K}$ para cada concentración de alpechín. Reactor con soporte Pansil.

\subsection{Comparación de los resultados experimen- tales con los obtenidos mediante el modelo de Chen-Hashimoto.}

Una vez determinados los valores de los parámetros cinéticos $\mu_{\max }$ y $\mathrm{K}$, se comparan los resultados experimentales con los que obtienen a partir de las ecuaciones propuestas por este modelo.

La ecuación que se utiliza para obtener los valores de $B$ es:

$$
B=B o\left(1-\frac{K}{\mu_{\max } \cdot \theta-1+K}\right)
$$

ecuación análoga a la (12).

En las tablas IX y $X$ se exponen los valores teóricos de $\mathrm{B}\left(\mathrm{ml}\right.$ de $\mathrm{CH}_{4}$ STP/g DQO añadido) en función del tiempo de retención (días) para las distintas concentraciones de alpechín, en los reactores con soporte Montmorillonita y Pansil, respectivamente.

Tabla IX

Valores teóricos de $B(\mathrm{ml} \mathrm{CH}$ STP/g DQO) en función del tiempo de retención (días) para las distintas concentraciones de alpechín. Reactor con soporte Montmorillonita.

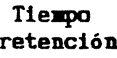

\begin{tabular}{|c|c|c|c|c|}
\hline & $10 \%$ & $20 \%$ & $40 \%$ & $80 \%$ \\
\hline 44,8 & 191,7 & 183,8 & 194,5 & 252,3 \\
\hline 32,0 & 185,6 & 179,0 & 191,0 & 249,2 \\
\hline 28,0 & 182,5 & 176,3 & 188,8 & 246,4 \\
\hline 22,4 & 176,0 & 169,8 & 183,6 & 233,3 \\
\hline 18,6 & 169,2 & 161,3 & 176,3 & 221,5 \\
\hline 11,2 & 149,5 & 144,9 & 164,3 & 220,5 \\
\hline
\end{tabular}

Concentración

Tabla $X$

Valores teóricos de $\mathrm{B}\left(\mathrm{ml} \mathrm{CH} \mathrm{CH}_{4}\right.$ STP/g DQO) en función del tiempo de retención (días) para las distintas concentraciones de alpechín. Reactor con soporte Pansil.

\section{Tiezpo}

retenclón

Concentración

\begin{tabular}{|c|c|c|c|c|}
\hline & $10 \%$ & $20 \%$ & $40 \%$ & $80 \%$ \\
\hline$\mu_{\max }$ (dias) & 0.14 & 0,11 & 0,09 & 0,07 \\
\hline$x$ & 0,41 & 0,29 & 0,15 & 0,05 \\
\hline
\end{tabular}

Parbetro

\section{Concentración}

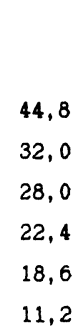

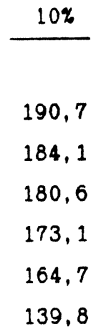

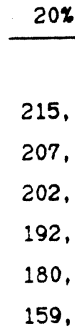

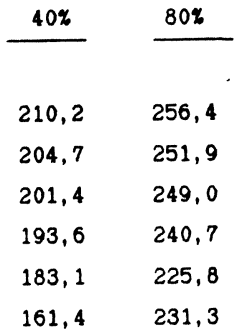


Como puede observarse comparando las tablas IX y X con la IV y V, respectivamente, existe un buen grado de ajuste entre los valores teóricos de $\mathrm{B}$ y los experimentales. Todos los valores experimentales de $\mathrm{B}$, correspondientes a los distintos tiempos de retención ensayados, para las diferentes concentraciones de alpechín en los dos reactores considerados, se desvían menos del $5 \%$ de sus valores teóricos. En concreto, un $91,6 \%$ de los puntos experimentales se desvían menos de un $4 \%$, un $87,5 \%$ se desvían menos de $3 \%$ y un $72,9 \%$ se separan menos de un $2 \%$ de sus valores teóricos obtenidos mediante la ecuación (15). Esto indica que el modelo ensayado predice el comportamiento de los reactores en todas las concentraciones de alpechín consideradas y los parámetros cinéticos obtenidos, representan la actividad de los microorganismos en el proceso contínuo de degradación anaerobia de las mismas.

\section{CONCLUSIONES}

1. Se ha comprobado que el modelo propuesto por Chen-Hashimoto es aplicable al proceso continuo de digestión anaerobia del alpechín; las ecuaciones obtenidas predicen el volumen de metano generado con un error inferior al 5\%; esto confirma que las constantes cinéticas obtenidas representan con fidelidad el comportamiento biocinético de los reactores.

2. Existe una considerable disminución de las constantes cinéticas $\mathrm{K}$ y $\mu_{\max }$ al aumentar la concentración de alpechín en los dos rectores considerados, lo que da idea de la inhibición que sufre el proceso.

3. La cuantía de esta inhibición depende del soporte utilizado; de esta forma al pasar de una concentración de alpechín del 10\% a una del $80 \%$ el parámetro $\mu_{\max }$ disminuye 4 y 2 veces en los reactores con soporte Montmorillonita y Pansil, respectivamente, mientras que la constante $\mathrm{K}$ disminuye 59,0 y 8,2 veces. Por tanto, en el Pansil la inhibición está mucho menos acentuada.
4. El coeficiente de rendimiento $\left(B_{o}\right)$ en el reactor con soporte Pansil es ligeramente superior al del reactor con Montmorillonita, lo que pone de manifiesto una vez más la influencia del soporte, sobre el proceso de digestión anaerobia de este agua residual.

\section{BIBLIOGRAFIA}

1. Borja, R. Martín, A. y Fiestas, J. A.- "Estudio cinético de la depuración anaerobia del alpechín en presencia de diversos soportes para la inmovilización de los microorganismos responsables del proceso".- Grasas y Aceites 41 (1990) 347356.

2. Borja, R. Martín, A. y Fiestas, J. A.- "Efecto de inhibición en el proceso de biometanización del alpechín en biorrectores con microorganismos inmovilizados en diversos tipos de soporte".- Grasas y Aceites 41 (1990) 397-403.

3. Rozzi, A.- "Estado del arte sobre la digestión anaerobia".Actas del $3^{\circ}$ Seminario de Depuración Anaerobia de aguas residuales, (1986) Valladolid.

4. Huysman, P. Van Meenen, P. Van Assche, P and Verstraete, W.- "Factors afeecting the colonization of non porous and porous packing materials in model upflow methane reactors".- Biotechnol. Lett 5 (1983) 643-648.

5. Murray, W. D. and Van den Berg, L.- "Effect of suppot material on the development of microbial fixed films converting acetic to methane".- J. Appl. Bacteriol, 51 (1981) 257-265.

6. Maestrojuan, Ma . Ga. Pérez, J. L. and Fiestas, J. A.- "A study of interaction between various materials used as supports and anaerobic bacterian.- Conference on anaerobic wastewater treatment AQUATECH, Amsterdam, The Netherlands (1986) 690-693.

7. Fiestas, J. A.- "La digestión anaerobia en la depuración de las aguas residuales de alta carga orgánican.- Actas II Congreso Mediterráneo de Ingeniería Química, (1981), Barcelona.

8. American Public Health Association (APHA).- "Standard Methods for the Examination of Water and Wastewater".- 16 th edition.- Washington, 1985.

9. Chen, Y. R. and Hashimoto, A. G.- "Kinetics of Methane fermentation".- Biotechnology and Bioengineering 8 (1978) 269-282. 